\title{
XIX. Ueber die chemische Zusammensetzung des Barytocalcits und des Alstonits.
}

\author{
Von
}

A. Becker in Leipzig.

Nach den wenigen vorhandenen Analysen des monoklinen Barytocalcits und des rhombischen Alstonits wurden diese beiden Mineralien als chemisch gleich zusammengesetzt $\left(\mathrm{BaCO}_{3}+\mathrm{CaCO}_{3}\right)$ angesehen, wobei indessen die Frage entstand, ob sie beide als is omorphe Mischungen von $\mathrm{BaCO}_{3}$ und $\mathrm{CaCO}_{3}$ zu betrachten seien oder ob nicht vielleicht bei einem derselben eine Molekularverbindung der beiden Carbonate von der Formel $\mathrm{BaCa} \mathrm{C}_{2} \mathrm{O}_{6}$ vorliege.

Auf Grund des von den analog zusammengesetzten Carbonaten abweichenden Krystallsystems des Barytocalcits und ferner auch, weil trotz der so ungeheuren Verbreitung des kohlensauren Kalkes diese Substanz noch nie monoklin krystallisirt beobachtet worden ist, meint $\mathrm{Groth}^{*}$ ), dass man es bei diesem Mineral mit einer derartigen Molekularverbindung zu thun habe, während der Alstonit eine isomorphe Mischung ungefähr äquivalenter Mengen beider Salze sei. Er findet eine Bestätigung seiner Ansicht in einer » merkwürdigen Beziehung zwischen den Krystallformen der beiden dimorphen Modificationen der salpetersauren Salze der einwerthigen Metalle einerseits und der Carbonate der zweiwerthigen Metalle andrerseits «, woruber er Näheres l. c. angiebt.

Um nun die Richtigkeit dieser Annahme experimentell zu prufen, habe ich eine Reihe von Analysen der betreffenden zwei Mineralien angestellt und will die Ergebnisse derselben hier mittheilen.

Leider gelang es mir nicht, wie ich gewünscht hätte, Material von verschiedenen Fundorten zu erhalten, sondern nur von Alston Moor ${ }^{* *}$ ) in Cum-

*) Tabellar. Uebersicht der Mineralien etc. 2. Aufl., 1882, S. 46 u. 47.

**) Nur ein Slück war von Hexham bei Fallowfeld in Northumberland, erwies sich aber als oberflachlich in $\mathrm{BaSO}_{4}$ umgewandelt und konnte daher nicht verwandt werden. 
berland und ich musste mich damit begnügen, mir wenigstens Stücke aus verschiedenen Sammlungen zu verschaffen, von welchen doch anzunehmen ist, dass sie nicht alle an derselben Stelle gebrochen worden siad. Einen Theil des Materials verdanke ich der Güte des Herrn Geh. Bergrath Prof. Dr. Zirkel, den Rest bezog ich von verschiedenen Händlern.

Das Aussuchen des Materials geschah mit möglichster Sorgfalt. Ich habe keine derben Stücke zur Analyse verwandt, sondern nur in Drusen frei aufsitzende, gut ausgebildete Krystalle, welche einzeln mit der Pincette abgebrochen und mit der Lupe auf Homogenität gepruft wurden, wobei die Alstonite, stets in spitzen, scheinbar hexagonalen Pyramiden auftretend, sich als fast ganz durchsichtig erwiesen, während die Barytocalcitkrystalle, meist vorwaltend am Ende verschieden ausgebildete Prismen zeigend, öfters nur durchscheinend waren. Nur die rein befundenen Krystalle gelangten zur Verwendung, die zweifelhaften wurden ausgeschieden. Die Reinheit des analysirten Materials ergiebt sich ubrigens auch daraus, dass beim Auflösen desselben in Salzsäure nur in drei Fällen und ein auch dann nur sehr geringer Rückstand blieb, welcher bei der qualitativen Untersuchung die Reaction auf Baryum und auf Schwefelsäure zeigte, also hauptsächlich aus $\mathrm{BaSO}_{4}$ bestand.

Als zweckmässigster Gang der Analyse ergab sich nach mancherlei Versuchen folgender: Nachdem durch spectralænalytische Untersuchung die totale Abwesenheit von Strontium nachgewiesen war, wurde zunächst die Kohlensäure nach bekannter Methode durch Ermittelung des Gewichtsverlustes nach Austreiben derselben durch Salzsäure bestimmt, dann aus der Lösung das Mangan durch Oxydation mittelst Chlorwasser als Manganperoxydhydrat gefällt, welches, stark gegluht, in gut wägbares Manganoxydul übergeht. Zur Trennung der nun noch zuruckbleibenden beiden Metalle Baryum und Calcium versetzte ich die salzsaure Lösung derselben mit schwefelsaurem und kohlensaurem Ammoniak und liess sie ]ängere Zeit (24 Stunden) bei gewöhnlicher Temperatur stehen, wobei $\mathrm{BaSO} \mathrm{O}_{4}$ und $\mathrm{Ca} \mathrm{C}_{3}$ niedergeschlagen werden; letzteres wurde wieder gelöst, vom $\mathrm{Ba}-$ rytsalz abfiltrirt und der Kalk dann in der gewöhnlichen Weise mit oxalsaurem Ammon bestimmt. Diese Trennungsmethode von $B a$ und $C a$ ist genauer, als die Fällung des $B a$ allein mit verdünnter Schwefelsäure, da hierbei stets etwas Kalksalz mit niedergerissen wird, welches sich durch Auswaschen nicht vollständig entfernen lässt.

Die Analysen ergaben folgende Resultate: 
1. Barytocalcit von Alston Moor, bezogen vom Mineralienhändler Stürtz in Bonn.

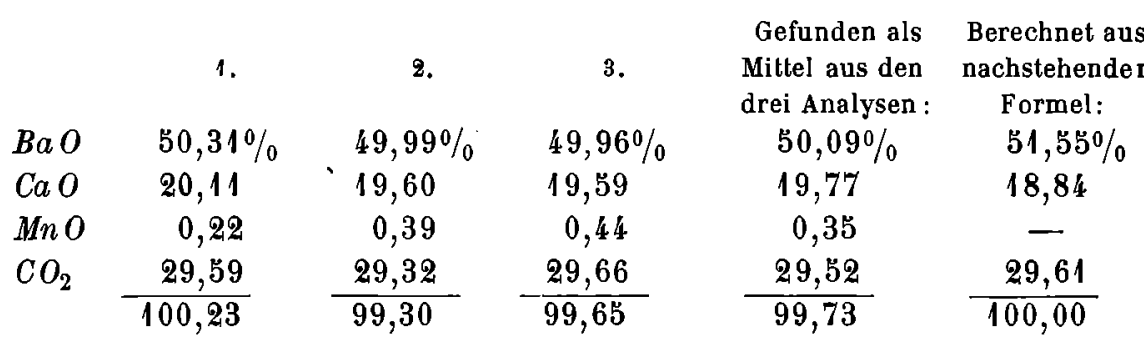

Berechnung :

Procente: Quotienten: Atomverhältniss:

$\begin{array}{lccc} & \text { Procente: } & \text { Quotienten: } & \text { Atomverhä } \\ B a & 44,85 & 0,3274 & 1 \\ C a & 14,12 & 0,3530 & 1,08 \\ C & 8,05 & 0,6708 & 2,05 \\ O & 32,98 & 2,0612 & 6,30\end{array}$

Diesen Verbältnisszahlen entspricht am besten die Formel:

$\mathrm{BaCO}_{3}+\mathrm{CaCO}_{3}$.

II. Barytocalcit von Alston Moor, bezogen vom Mineralienhändler Pech in Berlin.

1. 2. 3. 4. $\begin{gathered}\text { Gefunden als } \\ \text { Mittel aus den } \\ \text { vier Analysen: }\end{gathered} \begin{gathered}\text { Berechnet aus } \\ \text { nachstehender } \\ \text { Formel: }\end{gathered}$

\begin{tabular}{|c|c|c|c|c|c|c|}
\hline $\mathrm{BaO}$ & $50,02 \%$ & $51,36 \%$ & $49,58 \%$ & $50,50 \%$ & $50,36^{0} \%$ & $51,55 \%$ \\
\hline $\mathrm{CaO}$ & 19,66 & 17,68 & 20,40 & 19,13 & 19,22 & 18,84 \\
\hline$M n O$ & 0,30 & 0,23 & 0,30 & 0,18 & 0,25 & - \\
\hline $\mathrm{CO}_{2}$ & 29,32 & 29,46 & 29,36 & 29,61 & 29,44 & 29,61 \\
\hline Unl. Ruckst & 0,30 & 0,31 & 0,30 & 0,30 & 0,30 & - \\
\hline & 99,60 & 99,04 & 99,94 & 99,72 & 99,57 & 100 \\
\hline
\end{tabular}

Berechnung :

Procente: Quotienten: Atomverbältniss:

$\begin{array}{lccc} & \text { Procente: } & \text { Quotienten: } & \text { Atomverbä } \\ B a & 45,09 & 0,329 & 1 \\ C a & 13,79 & 0,343 & 1,04 \\ C & \mathbf{8 , 0 3} & 0,669 & \mathbf{2 , 0 3} \\ O & 33,16 & 2,072 & 6,30\end{array}$

Mithin ergiebt sich wiederum die Formel:

$$
\mathrm{BaCO} \mathrm{CaCO}_{3} \text {. }
$$


III. Barytocalcit von Alston Moor aus dem mineral. Museum der Universität Leipzig.

$\begin{array}{llc}\text { 1. 2. } & \begin{array}{c}\text { Gefunden als } \\ \text { Mittel aus den } \\ \text { drei Analysen : }\end{array} & \begin{array}{c}\text { Berechnet aus } \\ \text { nachstehender }\end{array} \\ \text { Formel : }\end{array}$

$\begin{array}{lccccc}\mathrm{BaO} & 50,81 \% & 52,89 \% & 51,07 \% & 51,59 \% & 51,55 \% \\ \mathrm{CaO} & 19,51 & 17,60 & 18,71 & 18,61 & 18,84 \\ \mathrm{MnO} & 0,38 & 0,32 & 0,34 & 0,35 & - \\ \mathrm{CO}_{2} & 29,41 & 29,60 & 29,15 & 29,39 & 29,61 \\ \text { Unl. Riuckst. } 0,18 & \frac{0,33}{100,74} & & \frac{0,32}{99,59} & \frac{0,28}{100,22} & \frac{1}{100,00}\end{array}$

Berechnung :

Procente: Quotienten: Atomverháltniss:

$\begin{array}{lrrl}\text { Ba } & 46,19 & 0,337 & 1,02 \\ C a & 13,29 & 0,332 & 1 \\ C & 8,02 & 0,668 & 2,01 \\ O & 32,50 & 2,030 & 6,11\end{array}$

Auch hier resultirt die Formel :

$$
\mathrm{BaCO}+\mathrm{CaCO} \text {. }
$$

IV. Alstonit von Alston Moor, vom Mineralienhändler Pech in Berlin bezogen, erstes Stúck.
1.
2. 3. Mittel aus den $\begin{array}{r}\text { Gerechnet aus } \\ \text { nachstehender }\end{array}$
2. 3. Mittel aus den nachstehender
2. 3. Mittel aus den $\begin{array}{r}\text { Gerechnet aus } \\ \text { nachstehender }\end{array}$ drei Analysen: Formel :

$\begin{array}{lccccc}\mathrm{BaO} & 45,26 \% & 43,96 \% & 44,86 \% & 44,69 \% & 46,34 \% \\ \mathrm{CaO} & 22,05 & 24,40 & 23,75 & 23,40 & 22,60 \\ \mathrm{MnO} & 0,27 & 0,30 & 0,30 & 0,29 & - \\ \mathrm{CO}_{2} & \frac{31,82}{99,40} & \frac{32,11}{100,77} & \frac{31,19}{100,10} & \frac{31,71}{100,09} & \frac{31,06}{100,00}\end{array}$

\section{Berechnung :}

Procente: Quotienten: Atomverhältniss :

$\left.\begin{array}{lrll}B a & 40,03 & 0,292 & 1 \\ C a & 16,71 & 0,418 & 1,43 \\ C & 8,48 & 0,707 & 2,42 \\ O & 34,78 & 2,174 & 7,44\end{array}\right\}=\left\{\begin{array}{r}3 \\ 4,29 \\ 7,26 \\ 22,32\end{array}\right.$

Diesen Verhältnissen kommt die Formel

$$
3 \mathrm{BaCO}_{3}+{ }_{4} \mathrm{CaCO}_{3}
$$

am nächsten. 
V. Alstonit von Alston Moor, vom Mineralienhändler Pech in Berlin bezogen, zweites Stück.

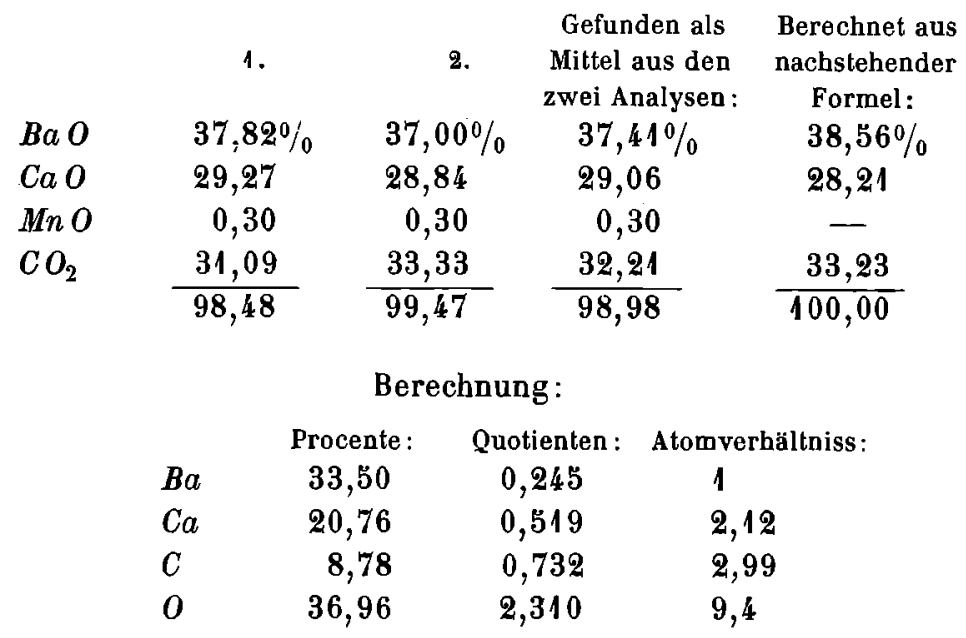

Diesen Zahlen entspricht die Formel

am besten.

$$
\mathrm{BaCO}+2 \mathrm{CaCO}_{3}
$$

\section{VI, Alstonit von Alston Moor, vom Mineralienhändler Gregory in London bezogen.}

\begin{tabular}{|c|c|c|c|c|c|}
\hline & 1. & 2. & 3. & $\begin{array}{l}\text { Gefunden als } \\
\text { Mittel aus den } \\
\text { drei Avalysen: }\end{array}$ & $\begin{array}{c}\text { Berechnet aus } \\
\text { nachstebender } \\
\text { Formel : }\end{array}$ \\
\hline$B a O$ & $51,01 \%$ & $51,33 \%$ & $50,58 \%$ & $50,97 \%$ & $51,55 \%$ \\
\hline $\mathrm{CaO}$ & 19,88 & 19,54 & 20,06 & 19,83 & 18,84 \\
\hline $\mathrm{CO}_{2}$ & 29,56 & 29,42 & 29,98 & 29,65 & 29,61 \\
\hline Unl. R & 0,25 & 0,25 & 0,25 & 0,25 & - \\
\hline & 100,70 & 100,54 & $1 \overline{00,87}$ & $\overline{100,70}$ & 100,00 \\
\hline
\end{tabular}

Berechnung :

Procente: Quotienten: Atomverbältniss:

$\begin{array}{lrll}B a & 45,64 & 0,333 & 1 \\ C a & 14,16 & 0,354 & 1,06 \\ C & 8,09 & 0,674 & 2,02 \\ O & 32,11 & 2,007 & 6,03\end{array}$

Mithin ergiebt sich die Formel :

$$
\mathrm{BaCO}+\mathrm{CaCO} \text {. }
$$


VII. Alstonit von Alston Moor aus dem mineralog. Museum der Universität Leipzig.

1. 2. 3. $\begin{array}{rr}\text { Gefunden als } & \text { Berechnet aus } \\ \text { Mittel aus den } & \text { nachstehender }\end{array}$

$\begin{array}{lccccc}\mathrm{BaO} & 52,19 \% & 51,87 \% & 50,28 \% & 51,45 \% & 51,55 \% \\ \mathrm{CaO} & 19,98 & 19,12 & 20,57 & 19,89 & 18,84 \\ \mathrm{MnO} & 0,20 & 0,20 & 0,20 & 0,20 & - \\ \mathrm{CO}_{2} & 29,45 & 29,66 & \frac{29,46}{100,51} & \frac{29,52}{101,06} & -\frac{29,61}{100,00}\end{array}$

Berechnung:

Procente: Quotienten: Atomverhältniss :

$\begin{array}{lrll}B a & 46,07 & 0,336 & 1 \\ C a & 44,21 & 0,355 & 1,05 \\ C & 8,05 & 0,671 & 2,00 \\ O & 31,67 & 1,980 & 5,89\end{array}$

Also resultirt wieder die Formel:

$$
\mathrm{BaCO}_{3}+\mathrm{CaCO}_{3} \text {. }
$$

Die hier gefundenen Zahlen fubren fur die drei untersuchten B a ry tocalcitdrusen auf die gleiche Formel: $\mathrm{BaCO}_{3}+\mathrm{CaCO}_{3}$, so dass dieses M ineral wohl als eine Molek u larverbind ung angesehen werden muss, während von den vier analysirten Alston it stucken; deren zwei zwar ebenfalls die gleiche Constitution: $\mathrm{BaCO} \mathrm{CO}_{3}+\mathrm{CaCO}_{3}$ besitzen, die beiden anderen aber, davon abweichend, die Formeln :

und

$$
\begin{array}{r}
3 \mathrm{BaCO} \mathrm{O}_{3}+{ }_{4} \mathrm{CaCO}_{3} \\
\mathrm{BaCO} \mathrm{CO}_{3}+2 \mathrm{CaCO} \mathrm{O}_{3}
\end{array}
$$

ergeben, sodass der Alstonit zweifellos eine is omorphe Mischung der beiden Carbonate ist.

Mithin wird durch die vorliegenden Untersuchungen die oben erwähnte Ansicht Groth's bestätigt.

Leipzig, den 25. Mai 1886. 